\title{
Depth-resolved degree of polarization of backscattered light and two- dimensional Mueller matrices of biological tissue measured by optical coherence tomography
}

Shuliang Jiao, Gang Yao, Lihong V. Wang

Shuliang Jiao, Gang Yao, Lihong V. Wang, "Depth-resolved degree of polarization of backscattered light and two-dimensional Mueller matrices of biological tissue measured by optical coherence tomography," Proc. SPIE 4257, Laser-Tissue Interaction XII: Photochemical, Photothermal, and Photomechanical, (9 July 2001); doi: 10.1117/12.434689

Event: BiOS 2001 The International Symposium on Biomedical Optics, 2001, San Jose, CA, United States 


\title{
Depth-resolved degree of polarization of backscattered light and two- dimensional Mueller matrices of biological tissue measured by optical coherence tomography \\ Shuliang Jiao, Gang Yao, Lihong V. Wang* \\ Optical Imaging Laboratory, Biomedical Engineering Program, Texas A\&M University, 3120 TAMU, College Station, Texas 77843-3120
}

\begin{abstract}
Mueller matrices provide a complete characterization of the optical polarization properties of biological tissue. A polarization-sensitive optical coherence tomography (OCT) system was built and used to investigate the optical polarization properties of biological tissues and other turbid media. The apparent degree of polarization (DOP) of the backscattered light was measured with both liquid and solid scattering samples. The DOP maintains the value of unity within the detectable depth for the solid sample while the DOP decreases with the optical depth for the liquid sample. Two-dimensional depthresolved images of the full Mueller matrices of biological tissues were measured with this system. These polarization measurements revealed some tissue structures that are not perceptible with standard OCT.
\end{abstract}

Keywords: OCT, Mueller matrix, Stokes vectors, polarization, degree of polarization(DOP)

\section{INTRODUCTION}

Optical coherence tomography (OCT) is an important noninvasive medical imaging modality, which can reveal subsurface structures of biological tissue. High spatial resolution $(\sim 1 \mu \mathrm{m})$ and high scanning speed (video rate) have been achieved over the past few years. ${ }^{1,2}$ Recently, polarization-sensitive OCT is emerging as a very attractive branch of OCT. ${ }^{3-8}$ Polarizationsensitive OCT can provide more information about the optical properties of biological tissue. Results of these studies revealed the importance of polarization as a contrast mechanism. As is known in polarimetry, Stokes vectors and Mueller matrices $^{9}$ provide complete representations of the polarization properties of light and optical samples, respectively. By measuring the Stokes vectors of the light backscattered from biological tissue and calculating the Mueller matrix from the Stokes vectors, one can obtain a complete characterization of the optical polarization properties of the tissue. With the combination of Mueller-matrix measurements with OCT, one can obtain the Mueller matrix of a sample with OCT resolution. Yao and $\mathrm{Wang}^{7}$ first reported the measurement of two-dimensional depth-resolved Mueller matrices of biological tissue with OCT. Jiao et $\mathrm{al}^{8}$ further reported the experiment results with the same experimental setup. The results revealed some structures that were not observable in standard OCT. Therefore, Mueller matrices provide a unique approach to characterizing biological tissue.

In this paper we give a detailed account of our novel polarization-sensitive OCT system for characterizing the polarization properties of biological tissue and other turbid media with both depth and lateral resolution. A succinct relationship between the Stokes vectors and the Mueller matrix in our measurement approach was derived. We measured the Stokes vectors of backscattered light from solid biological tissue and liquid turbid media. The apparent degree of polarization was calculated from the Stokes vectors. The calculated results revealed the difference between solid and liquid in the degree of polarization as a function of the optical depth. An explanation for the mechanism contributing to this difference was speculated. We also measured 2D images of the full $4 \times 4$ Mueller matrix of a piece of rat bone.

\section{STOKES VECTORS AND MUELLER MATRICES}

In polarimetry, the Stokes vector $\mathbf{S}$ of a light beam is constructed based on six flux measurements with different polarization analyzers in front of the detector:

\footnotetext{
* To whom all correspondence should be addressed. Tel: 979-847-9040; Fax: 979-845-4450; Email: LWang@ tamu.edu; URL: http://oilab.tamu.edu.
} 


$$
S=\left(\begin{array}{l}
S_{0} \\
S_{1} \\
S_{2} \\
S_{3}
\end{array}\right)=\left(\begin{array}{l}
I_{H}+I_{V} \\
I_{H}-I_{V} \\
I_{P}-I_{M} \\
I_{R}-I_{L}
\end{array}\right)=\left(\begin{array}{l}
I_{H}+I_{V} \\
I_{H}-I_{V} \\
2 I_{P}-I_{H}-I_{V} \\
2 I_{R}-I_{H}-I_{V}
\end{array}\right),
$$

where $I_{H}, I_{V}, I_{P}, I_{M}, I_{R}$, and $I_{L}$ are the light intensities measured with a horizontal linear polarizer, a vertical linear polarizer, a $+45^{\circ}$ linear polarizer, a $-45^{\circ}$ linear polarizer, a right circular analyzer, and a left circular analyzer in front of the detector, respectively. Because of the relationships $I_{H}+I_{V}=I_{P}+I_{M}=I_{R}+I_{L}=I$, where $I$ is the intensity of the light beam measured without any analyzer in front of the detector, a Stokes vector can be determined by four independent measurements, for example, $I_{H}, I_{V}, I_{P}$, and $I_{R}$.

From the Stokes vector, the degree of polarization (DOP), the degree of linear polarization (DOLP), and the degree of circular polarization (DOCP) are derived as:

$$
D O P=\frac{\sqrt{S_{1}^{2}+S_{2}^{2}+S_{3}^{2}}}{S_{0}}, D O L P=\frac{\sqrt{S_{1}^{2}+S_{2}^{2}}}{S_{0}}, D O C P=\frac{S_{3}}{S_{0}} .
$$

The Mueller matrix (M) of a sample transforms an incident Stokes vector $\mathbf{S}_{i n}$ into the corresponding output Stokes vector $\mathbf{S}_{\text {out }}$.

$$
\mathbf{S}_{\text {out }}=\mathbf{M S}_{\text {in }}
$$

The Mueller matrix can be experimentally obtained by measurements with different combinations of source polarizers and detection analyzers. We may express the $4 \times 4$ Mueller matrix as $\mathbf{M}=\left[\mathbf{M}_{0} \mathbf{M}_{1} \mathbf{M}_{2} \mathbf{M}_{3}\right]$, where $\mathbf{M}_{0}, \mathbf{M}_{1}, \mathbf{M}_{2}$, and $\mathbf{M}_{3}$ are four column vectors of four elements each. The four output Stokes vectors corresponding to the four incident polarization states $H, V, P$, and $R$ are denoted respectively by $\mathbf{S}_{H}, \mathbf{S}_{V}, \mathbf{S}_{P}$, and $\mathbf{S}_{R}$. These four output Stokes vectors are experimentally measured based on Eq. (1) and can be expressed as:

$$
\left\{\begin{array}{l}
\mathbf{S}_{H}=M_{H i}=M_{0}+M_{1} \\
S_{V}=M S_{V i}=M_{0}-M_{1} \\
S_{P}=M S_{P i}=M_{0}+M_{2} \\
S_{R}=M S_{R i}=M_{0}+M_{3}
\end{array} .\right.
$$

The Mueller matrix can then be calculated from the output Stokes vectors:

$$
\mathbf{M}=\frac{1}{2}\left[\mathbf{S}_{H}+\mathbf{S}_{V}, \mathbf{S}_{H}-\mathbf{S}_{V}, 2 \mathbf{S}_{P}-\mathbf{S}_{H}-\mathbf{S}_{V}, 2 \mathbf{S}_{R}-\mathbf{S}_{H}-\mathbf{S}_{V}\right]
$$

In other words, at least four independent Stokes vectors for different source polarization states must be measured to determine a full Mueller matrix, where each Stokes vector requires four independent intensity measurements with different analyzers.

\section{EXPERIMENTAL SETUP}

A schematic of the OCT system for our studies is shown in Fig. 1. A superluminescent diode (SLD) laser with a center wavelength of $850 \mathrm{~nm}$ and a FWHM bandwidth of $26 \mathrm{~nm}$ is used as the light source. After passing through the polarizer P, the half-wave plate HW, and the quarter-wave plate QW, the light is split by a nonpolarization beam splitter (NBS). The sample beam is focused into the sample by an objective lens (L1) with an N.A. of 0.15 . The reference beam passes through a variable-wave plate and is reflected back. The reflected beams from the reference and sample arms are coupled into a singlemode fiber and detected by a silicon photodiode. The minimal detectable signal of the system is $-100 \mathrm{~dB}$. A single depth scan through $1.5 \mathrm{~mm}$ takes 6 seconds in the current system. The depth resolution of this system is $10 \mu \mathrm{m}$. The focal spot size of the objective lens (L1) is $6.9 \mu \mathrm{m}$ in air and is larger in tissue. The lateral resolution is expected to be around $10 \mu \mathrm{m}$.

We achieve four different incident polarization states $H, V, P$, and $R$ by rotating the half-wave plate (HW) and the quarter-wave plate $(\mathrm{QW})$ in the source arm. For each of these four incident polarization states, the variable-wave plate (VW) at the reference arm is adjusted to sequentially achieve the $H, V, P$, and $R$ polarization states. A total of 16 polarization- 
sensitive OCT images are acquired and processed to obtain the 16 Mueller matrix images $\left[M_{i j}\right]$. Alternatively, if the Stokes vector of the backscattered light is sought for a given incident polarization state, only four measurements need to be acquired by varying the reference polarization state. The Stokes vector is then calculated based on Eq. (1).

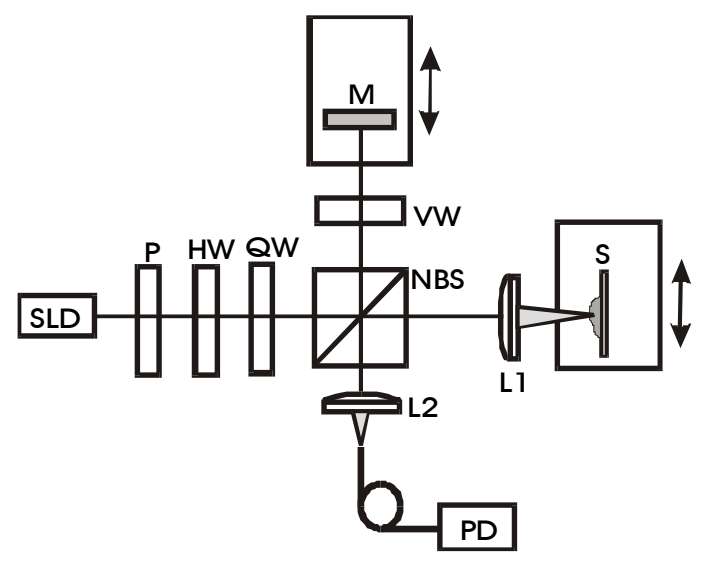

Fig.1 Schematic of the polarization-sensitive OCT system

Because the beam splitter is not an ideal polarization-independent optical element, the Mueller matrix of the beam splitter was measured for calibration. The Mueller matrices of simple optical elements measured with our setup agree with their known ideal matrices to within an error of $5 \%-10 \%$ after calibration with the measured Mueller matrix of the beam splitter.

\section{RESULTS AND DISCUSSION}

We measured the Stokes vector $\mathbf{S}_{H}$ of a 5\% Intralipid solution and a piece of bone tissue from a fish with the incident light in a state of horizontal linear polarization $(H)$. The Stokes vectors were then used to calculate the DOP for the sample. The results are shown in Figs. $2 \&$ 3, where the optical depth means the product between physical depth and the refractive index of the sample. Figure 2 shows the $S_{H 0}$ and DOP for the 5\% Intralipid solution; Figure 3 shows the $S_{H 0}$ and DOP for the bone sample. In figure (3), the whole measurement depth of $500 \mu \mathrm{m}$ are divided into 5 segments each of which is $100 \mu \mathrm{m}$ in length and has 200 points. DOP is calculated for each point. The mean and the standard deviation for each segment are then calculated and plotted in the figure as the center value of each segment. All the data were averaged over 20 scans. Polynomial fitting was applied to the data for the Intralipid solution to reduce the fluctuation before calculating the Stokes vector.

The difference in the degree of polarization is striking between the liquid and the solid samples. For the liquid sample, the DOP decreases as the optical depth increases as shown in Fig. 2. For the solid sample, however, the DOP is approximately unity throughout the detectable range of optical depth apart from the fluctuation as shown in Fig. 3. The fluctuation of the DOP around unity for the solid sample is likely caused by the anisotropy of optical properties of the sample, which was not observed in the isotropic liquid samples. Anisotropy such as birefringence would cause the same physical feature to appear at different optical depths when it is measured with different analyzing polarization states. Conversely, the four quantities measured with different analyzing polarization states at a given optical depth may correspond to signals from slightly different physical depths. It would be challenging to accurately align the physical features among the one-dimensional depthscan images of different analyzing polarization states. This slight misalignment causes the fluctuation of DOP.

The salient difference in degree of polarization between the liquid and solid samples indicates that a liquid medium acts upon our OCT signals differently from a solid medium. The variation of DOP with the optical depth and the solution concentration signifies that the apparent depolarization effect in liquid increases with the depth and the concentration. In principle, a DOP of less than unity means that the detected backscattered light is partially depolarized. Due to scattering, the completely polarized incident light is converted into non-uniformly polarized scattered light. If conventional intensity-based measurements were employed to detect the polarization property of this non-uniformly polarized light, the DOP would be less than unity and decrease with increasing scattering. The reduction of DOP is because that the light impinging upon different locations on the analyzers in front of the detector has different polarization states and adds in intensity after passing through the analyzers. The intensity signals of the light from different locations measured with orthogonal analyzers will partially offset each other in the calculation of the Stokes vector while the total intensity measured without analyzers is always the sum of the light from all of the locations. 


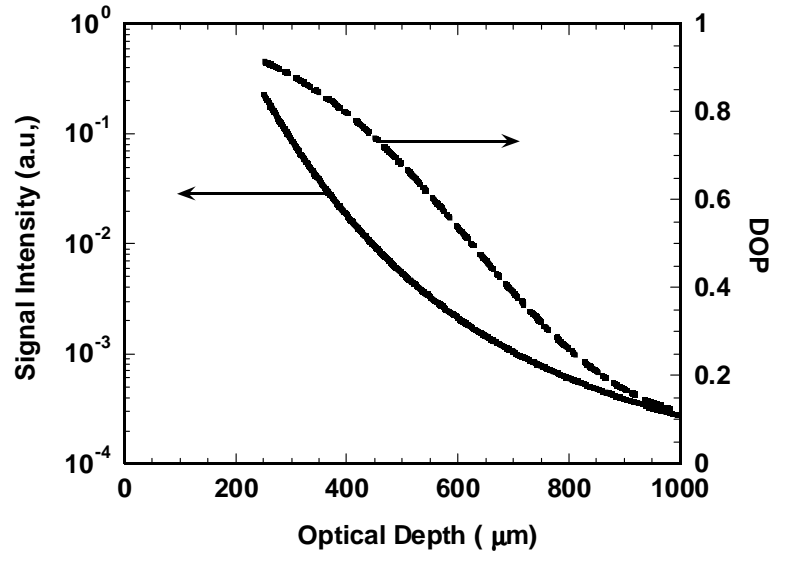

Fig.2 $S_{H 0}$ and DOP for 5\% Intralipid solution.

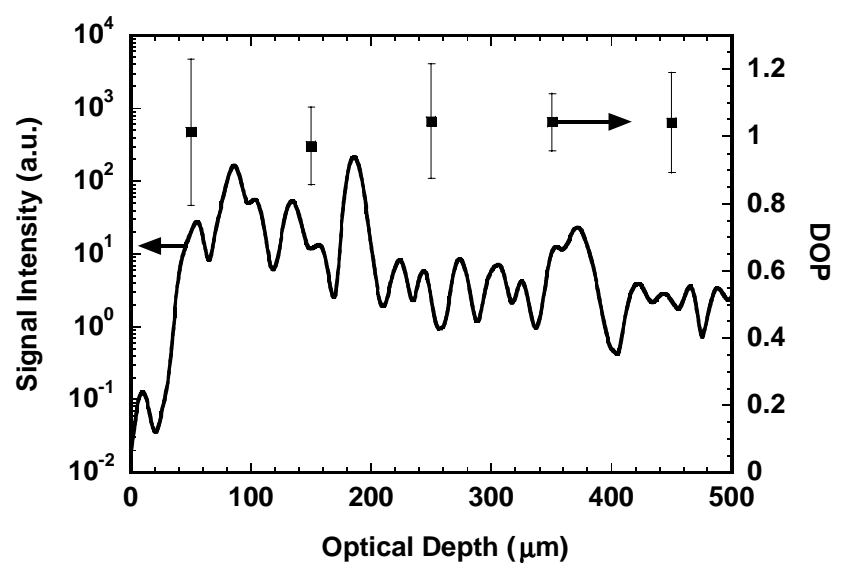

Fig. $3 S_{H 0}$ and DOP for a fish bone tissue.

However, OCT is an amplitude-based detection system by interference heterodyne. OCT detects the electric field of only the coherent part of the backscattered light. As is shown in Eq. (6), the electric field of the light from different locations of the detector is projected onto the analyzing polarization state $\mathbf{E}_{\mathbf{r}}$, then added in amplitude. Equivalently, the electric field of the light from different locations of the detector is summed in vector, and the vector sum $\mathbf{E}_{\mathbf{s}}$ is then projected onto the analyzing polarization state. Because of this coherent detection scheme in OCT, a DOP of unity is maintained despite scattering as observed in the solid sample. Therefore, the conventional depolarization process in intensity-based measurements does not account for the decrease of DOP in the liquid media.

$$
\begin{aligned}
I & =\mathbf{E}_{\mathrm{r}} \cdot \mathbf{E}_{\mathrm{s} 1}+\mathbf{E}_{\mathrm{r}} \cdot \mathbf{E}_{\mathrm{s} 2}+\mathbf{E}_{\mathrm{r}} \cdot \mathbf{E}_{\mathrm{s} 3}+\ldots \\
& =\mathbf{E}_{\mathrm{r}} \cdot\left(\mathbf{E}_{\mathrm{s} 1}+\mathbf{E}_{\mathrm{s} 2}+\mathbf{E}_{\mathrm{s} 3}+\ldots\right) \\
& =\mathbf{E}_{\mathrm{r}} \cdot \mathbf{E}_{\mathrm{s}} \\
\mathbf{E}_{\mathrm{s}} & =\mathrm{E}_{\mathrm{s} 1}+\mathbf{E}_{\mathrm{s} 2}+\mathbf{E}_{\mathrm{s} 3}+\ldots
\end{aligned}
$$

We speculate that the decrease of the apparent DOP in liquid is caused by the Brownian motion of the scattering particles and the signal averaging in the data acquisition. Brownian motion causes the polarization state of the backscattered light to fluctuate around an average state. Because our OCT system converts the interference fringes into an envelope of rectified fringes, only this positive envelope is detected and averaged.

To illustrate this point, we let $I_{H}, I_{V}, I_{P}$, and $I_{R}$ denote the intensities of the average polarization state analyzed by horizontal linear polarization, vertical linear polarization, $+45^{\circ}$ linear polarization, and right circular polarization state, respectively. Let $I_{n}$ denote the average intensity caused by the Brownian fluctuation, which is assumed to be the same for all of the four measurements with different analyzers for simplicity. The measured Stokes vector can be expressed as:

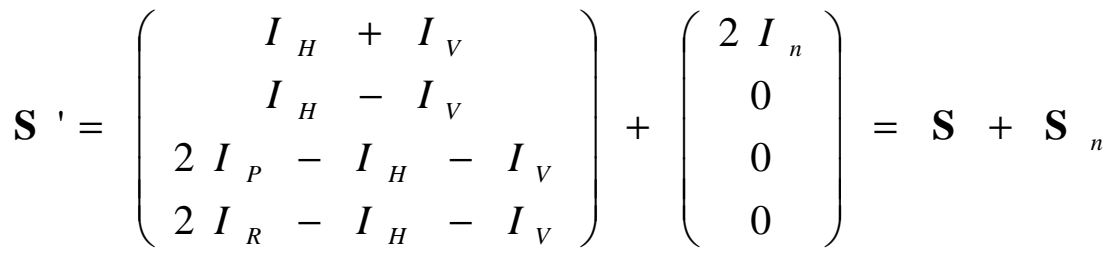

The DOP of $\mathbf{S}$ is unity while the DOP of $\mathbf{S}_{n}$ is zero. The DOP of $\mathbf{S}^{\prime}$ is

$$
\operatorname{DOP}\left(\mathbf{S}^{\prime}\right)=\operatorname{DOP}(\mathbf{S}) \frac{S_{0}}{S_{0}+2 I_{n}}=\frac{S_{0}}{S_{0}+2 I_{n}}
$$

As can be seen, the apparent DOP of the measured Stokes vector is less than unity. The increase of Intralipid concentration means an increase of random scattering that the light encounters per unit optical depth. An increase in optical depth means that the backscattered light encounters more scattering events. The increased scattering events would cause more fluctuation because each scattering event has Brownian motion. Therefore, the average intensity $I_{n}$ would increase with both the optical depth and scatterer concentration in liquid, which would accordingly decrease the apparent DOP. This hypothesis 
can be ultimately tested if our setup is improved such that the Stokes vector of a liquid sample can be measured in a sufficiently short time period.

We measured the 2D Mueller-matrix images of a piece of rat bone. The raw images and the images of the corresponding 16 Mueller-matrix elements are shown in Fig. 4. The four Stokes vectors corresponding to the four incident polarization states were first calculated using Eq. (2) and then were used to calculate the Mueller matrix. The image size is $1.0 \mathrm{~mm}$ in optical depth and $0.5 \mathrm{~mm}$ in the lateral dimension. The $1.0 \mathrm{~mm}$ optical depth may be converted to approximately $0.67 \mathrm{~mm}$ in physical depth assuming the index of refraction of the bone sample is 1.5. The image of the Mueller-matrix element $M_{00}$ corresponds to a polarization-independent image as acquired by a nonpolarization OCT system. The other Mueller-matrix elements $M_{i j}$ are pixel-wise normalized by $M_{00}$ image. The polarization-independent element $M_{00}$ reveals significantly less information than the other elements as clearly shown in the figure. Strong layered structures are clearly seen in some of the images such as $M_{22}, M_{23}, M_{32}$, and $M_{33}$.

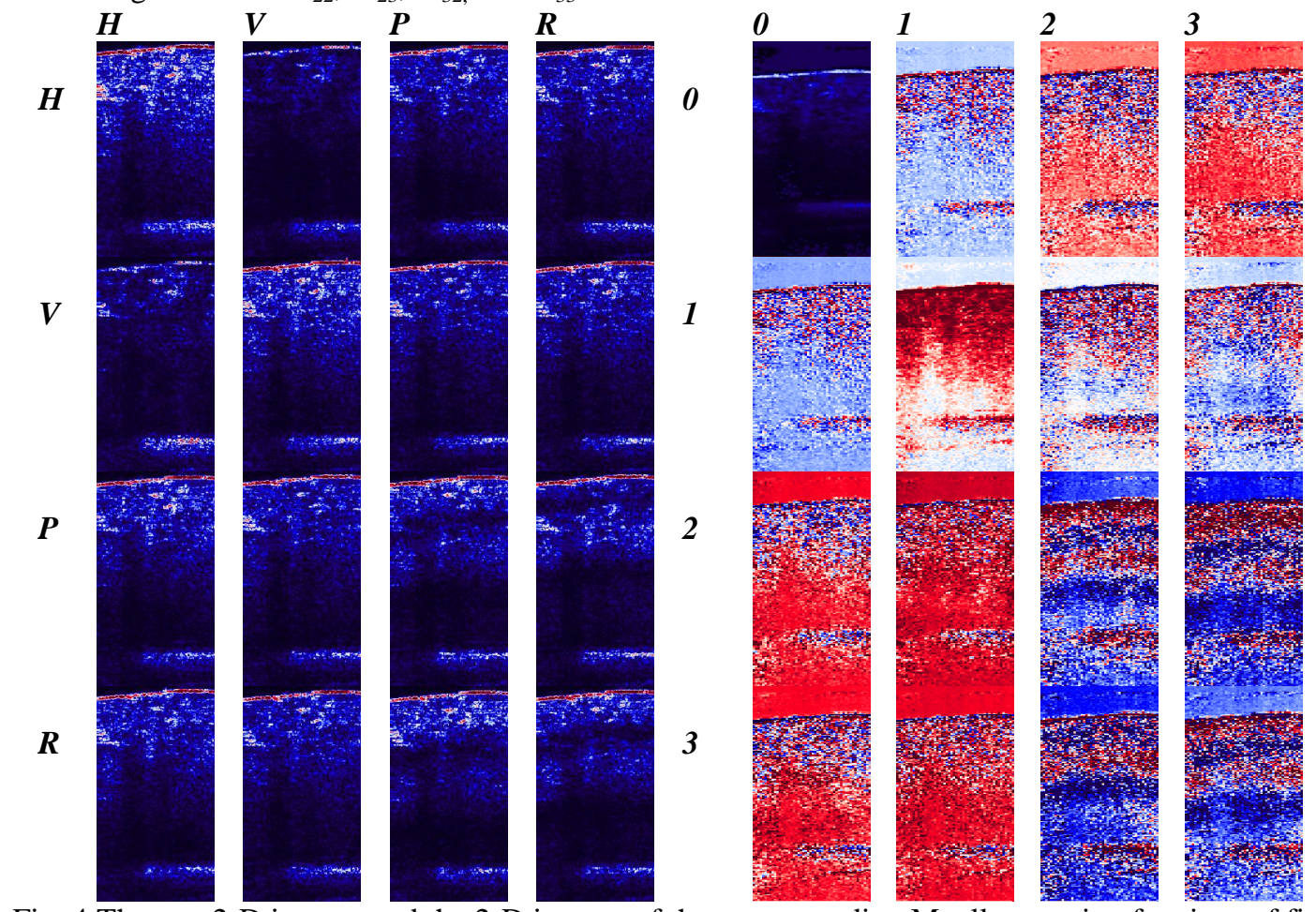

Fig. 4 The raw 2-D images and the 2-D images of the corresponding Mueller matrix of a piece of fish bone

\section{CONCLUSION}

In summary, we demonstrated a novel imaging technique that measures Stokes vectors and Mueller matrices with optical coherence tomography in biological tissue or other turbid media. With this technique we measured the DOP, DOLP, and DOCP of liquid and solid samples. The DOP maintains the value of unity within the detectable depth for the solid sample while the DOP decreases with the optical depth for the liquid sample. This different behavior of liquid is probably related to the Brownian motion of the scattering particles in liquid. The Brownian motion of the particles causes random variation of the polarization state of the incident light. The 2D images of the Mueller matrices revealed more information about the tissue samples than standard OCT. The Mueller-matrix imaging system has potential applications in both scientific research and clinical practice. 


\section{ACKNOWLEDGEMENTS}

Thanks to Dr. G. Stoica, College of Veterinary Medicine, Texas A\&M University for providing the sample. This study was sponsored in part by National Institutes of Health grants R29 CA68562, R01 CA71980, and R21 CA83760, by National Science Foudation grant BES-9734491, and by Texas Higher Education Coordinating Board grant 000512-0123-1999.

\section{REFERENCES}

1. W. Drexler, U. Morgner, F. X. Kärtner, C. Pitris, S. A. Boppart, X. D. Li, E. P. Ippen, and J. G. Fujimoto, “In vivo ultrahigh-resolution optical coherence tomography," Opt. Lett. 24, 1221 (1999).

2. A. Rollins, S. Yazdanfar, M. Kulkarni, R. Ungarunyawee, and J. Izatt, "In vivo video rate optical coherence tomography," Opt. Exp. 3, 219 (1998), www.osa.org.

3. M. J. Everett, K. Schoenenberger, B. W. Colston, Jr., and L. B. Da Silva, "Birefringence characterization of biological tissue by use of optical coherence tomography," Opt. Lett. 23, 228 (1998).

4. J. F. de Boer, S. M. Srinivas, A. Malekafzali, Z. Chen and J. S. Nelson, "Imaging thermally damaged tissue by polarization sensitive optical coherence tomography," Opt. Exp. 3, 212 (1998), www.osa.org.

5. J. M. Schmitt and S. H. Xiang, "Cross polarized backscatter in optical coherence tomography of biological tissue," Opt. Lett. 23, 1060 (1998).

6. J. F. de Boer, T. E. Milner and J. Stuart Nelson, "Determination of the depth-resolved Stokes parameters of light backscattered from turbid media by use of polarization-sensitive optical coherence tomography," Opt. Lett. 24 , 300 (1999).

7. G. Yao and L. V. Wang, "Two dimensional depth-resolved Mueller matrix characterization of biological tissue by optical coherence tomography," Opt. Lett. 24, 537 (1999).

8. S. Jiao, G. Yao and L Wang, "Depth-resolved two-dimensional Stokes vectors of backscattered light and Mueller matrices of biological tissue measured with optical coherence tomography," Appl. Opt. 34, 6318(2000)

9. W. S. Bickel and W. M. Bailey, "Stokes vectors, Mueller matrices, and polarized scattered light," Am. J. Phys. 53, 468478(1995). 